\title{
HLA Matching in Unrelated Stem Cell Transplantation up to Date
}

\author{
Daniel Fürst ${ }^{a, b} \quad$ Christine Neuchel $^{a, b} \quad$ Chrysanthi Tsamadou $^{a, b}$ \\ Hubert Schrezenmeier ${ }^{a}$ b Joannis Mytilineos ${ }^{a, b}$ \\ anstitute of Clinical Transfusion Medicine and Immunogenetics UIm, German Red Cross Blood Transfusion Service \\ Baden-Württemberg-Hessen, and University Hospital UIm, Ulm, Germany; ${ }^{b}$ Institute of Transfusion Medicine, \\ University of Ulm, Ulm, Germany
}

\section{Keywords}

HLA matching · Stem cell transplantation · Bone marrow transplantation · Unrelated donor

\begin{abstract}
Unrelated hematopoietic stem cell transplantation (HSCT) has evolved from an experimental protocol to a potentially curative first-line treatment in certain disease instances. Factors enabling this transformation were the optimization of treatment protocols and supportive care as well as the availability of a large number of donors worldwide along with the higher quality and reliability of HLA typing. The main criterion for donor selection is HLA compatibility. In this review we discuss the current clinical evidence of HLA matching in unrelated HSCT. In this context, we address methodical aspects of transplantation immunobiology research and discuss the impact of locus and resolution of HLA differences. Furthermore, we address special constellations such as unidirectional mismatches or the presence of nonexpressed alleles as well as HLA alloimmunization and describe the perspective for HLA typing and matching strategies in the future, given the implementation of novel complete or near-complete gene typing approaches using next-generation sequencing short read technology, which are now entering the standard of clinical care.
\end{abstract}

(c) 2019 S. Karger AG, Basel

\section{KARGER}

(ㄷ) 2019 S. Karger AG, Basel

\section{Introduction}

Overcoming the histocompatibility barrier for the transplantation of organs and tissues from allogeneic donors is a major achievement of modern medicine. Crucial for this development was the discovery of HLA and the description of its function and structure within the framework of acquired immunity $[1,2]$. The transplantation of blood stem cells represents a massive interference with the immunological homeostasis of patients. A new lympho-hematopoietic system emerges in the recipient from donor stem cells, which replaces the diseased bone marrow. Immunological reactions of patient against donor cells are referred to as host-versus-graft responses (HvG vector). Immune reactivity of donor cells against recipient tissue is referred to as graft-versus-host ( $\mathrm{GvH}$ vector) alloreactivity, which exerts an immunotherapeutic effect on neoplastic cells called graft-versus-leukemia (GvL) effect. However, GvH alloreactivity is also associated with severe side effects in transplant recipients [3]. The latter is called $\mathrm{GvH}$ disease (GvHD), which is a major factor in transplant-related morbidity and mortality and affects a large proportion of patients in various degrees of severity [4]. The indications for allogeneic stem cell transplantation are broad and include various malignant and benign diseases of the hematopoietic system, encompassing aplastic anemia, hemoglobinopathies, inborn errors of metabolism, and severe immunodeficiencies [5, 6]. Of eminent importance is the histocompatibility between donor and recipient [7]. Over the past two to three decades, allogeneic stem cell transplantation has evolved 
from an experimental therapy to a standardized procedure. Conditioning treatments have been optimized, which makes transplantation safer, particularly for elderly patients and those with comorbidities [8]. Due to the continuously growing number of well-typed volunteer donors worldwide, more and more patients can be provided with a suitable donor when no matched family donor is available [9]. Also, the transition from serological to high-resolution molecular HLA typing (i.e., unambiguous typing of the antigen-binding site of HLA alleles formed by the $a 1 / \alpha 2$ domains, encoded by exons 2 and 3 for HLA class I, and the $\alpha 1$ domain encoded by exon 2 for HLA class II, including exclusion of nonexpressed variants) has vastly improved the results of allogeneic hematopoietic stem cell transplantation (HSCT) [10-12]. The most important immunogenetic factors recognized so far are the classical HLA genes (HLA-A, HLA-B, HLA-C, HLA-DRB1, HLA-DQB1, and HLA-DPB1) [12]. In this review we summarize the current evidence in HLA matching and donor selection for allogeneic matched unrelated stem cell transplantation (i.e., $8 / 8$ for the loci HLA-A, HLA-B, HLA-C, and HLA-DRB1 according to US standards and 10/10 for the loci HLA-A, HLA-B, HLA-C, HLA-DRB1, and HLA-DQB1 according to European standards) and give a perspective on possible future developments given the introduction of novel nextgeneration sequencing-based typing approaches $[10,12]$.

\section{Methodical Aspects of the Investigation of HLA Incompatibility Studies}

Up to date 23,907 HLA alleles have been described (HLA Database 3.37 2019-07) [13]. The resulting diversity of HLA phenotypes complicates the investigation of the impact of HLA differences on the outcome of HSCT. HLA incompatibilities occur at different loci, with different allele combinations, and in different HLA phenotype contexts, which in turn affect the spectrum of the presentable antigens [14]. Basically, each HLA phenotype is unique, and grouping in scientific analyses is a simplification that yields a value averaged over many instances. In addition, qualitative and quantitative effects must be considered in such analyses. Quantitative effects are usually reported by estimates of regression methods (Cox regression, competing risks regression) and as relative risk or hazard ratio of events in one group compared to the control or reference group. Qualitative effects are examined by the analysis of different endpoints. Important endpoints in this context are overall survival, disease-free survival, transplant-related mortality (TRM), relapse incidence, nonrelapse mortality and GvHD incidences [15]. As a result of the fact that the majority of patients are transplanted with completely matched donors (accord- ing to the current criteria), very small groups are found in subanalyses according to mismatched locus or allele combinations. This in turn limits the statistical power. Consequently, the size of the study cohorts is an important factor. In order to investigate HLA matching in unrelated HSCT, larger cohort sizes are often necessary, which can only be achieved with retrospective and cross-sectional studies using registry data. Furthermore, ethical considerations prevent controlled trials with study arms selecting HLA-mismatched donors over HLA-matched donors when inferior survival is expected for the patients with mismatched donors. Therefore, the level of evidence available in the literature is usually $2 \mathrm{~b}$ or less (level $2 \mathrm{~b}$ : individual cohort study or retrospective cohort study). Nevertheless, the HLA matching grade is one of the most important predictors for the success of HSCT and usually the most important criterion for selection of unrelated donors.

\section{The Impact of HLA-A and HLA-B Mismatches}

Classical HLA molecules are codominantly expressed, with HLA-A and HLA-B showing the highest expression among HLA class I molecules [16]. In all major studies, HLA-A and HLA-B mismatches increased the risk for mortality (Table 1). In large retrospective studies, mostly based on patients with malignant primary disease, relative risk ratios in the range of 1.17-2.20 and 1.16-1.90 have been reported for single HLA-A and HLA-B mismatches, respectively, when compared to patients with completely matched donors. In practice this corresponds to one or two additional deaths in 10 treated patients solely due to HLA incompatibility [7, 17-21]. In the presence of more than one HLA incompatibility, the risk of individual mismatched loci cumulates, so that a careful benefit/risk evaluation should be carried out in these patients, taking into account alternative stem cell sources, such as haploidentical stem cell transplantation or umbilical cord blood (CB) transplantation [21, 22]. To date, no significant quantitative difference between HLA antigen incompatibilities, at the level of serologically distinguishable specificities, and HLA allele differences for these loci has been shown in any of the mentioned studies. Alloreactivity in HSCT is dominated by T lymphocytes, which are present in the transplant and are newly formed from donor stem cells [23]. B cells reconstitute later after transplantation, and their influence on acute GvHD is considered less important, albeit conclusive data on this aspect are sparse [24]. High-resolution HLA typing in HSCT is mandatory as relevant $\mathrm{T}$ cell epitopes cannot be differentiated in the region of the peptide-binding domains of HLA molecules by low-resolution HLA typing [25]. The occurrence of GvHD correlates strongly with the pres- 

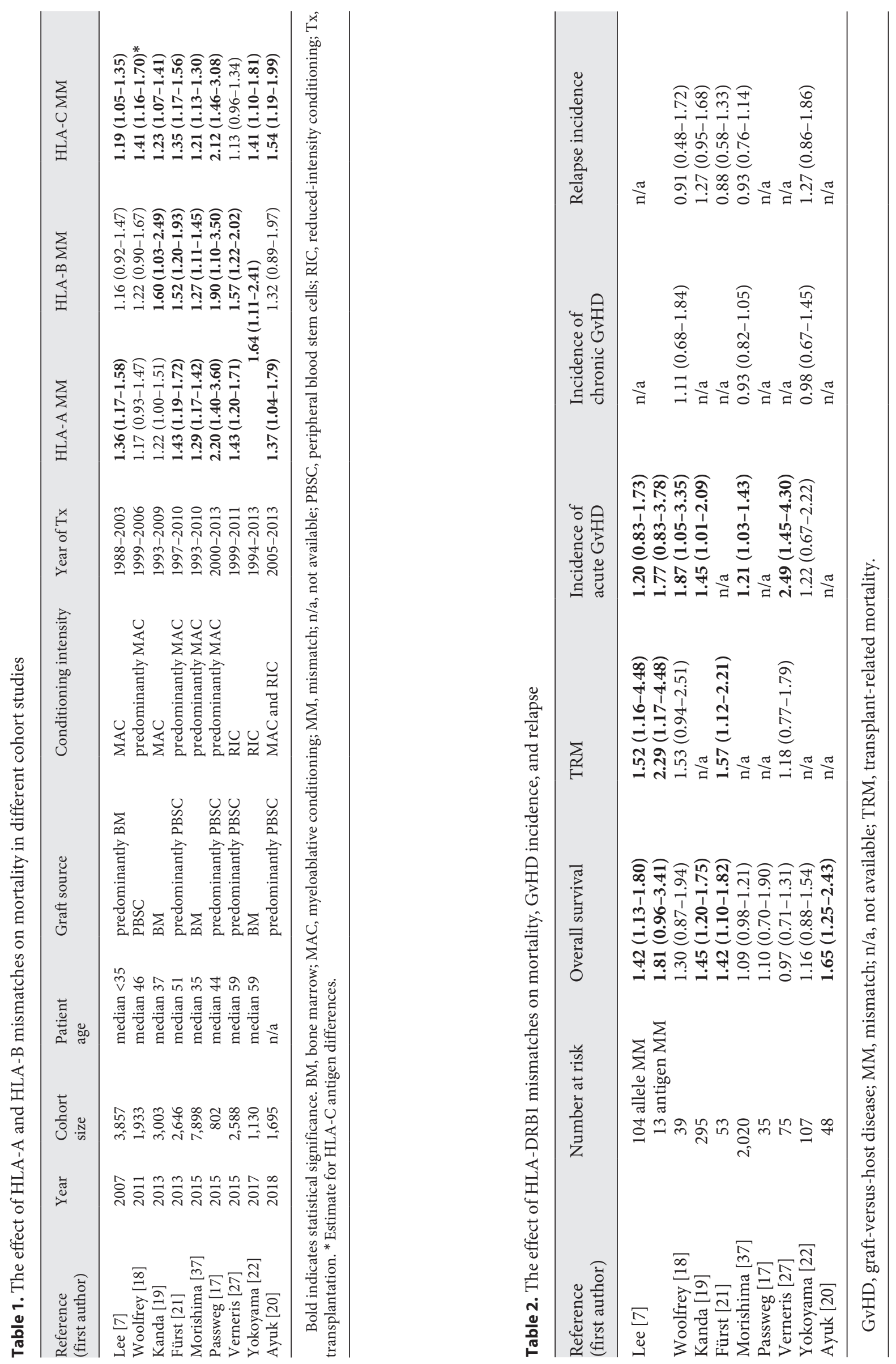
ence of HLA incompatibilities [26]. Since GvHD is one of the main causes of morbidity after blood stem cell transplantation, HLA mismatches also have an effect on TRM. Here the relative risks lie in the range of 1.07-2.26 in the presence of a single HLA-A or HLA-B difference [17, 21]. Although GvHD is perceived to be accompanied by a positive graft-versus-leukemia component, a lower but not significantly reduced incidence of disease recurrence in single mismatched transplantations has been observed in several large studies [7, 18, 21, 27]. Consequently, the HLA effect on disease-free survival is less pronounced since here the occurrence of a disease relapse is also evaluated. It can be concluded that the unfavorable effect of HLA incompatibilities on TRM overcomes a potentially beneficial effect on disease recurrence. The latter should therefore not be included in considerations on the selection of donors in the presence of HLA-A and HLA-B mismatches. It remains to be clarified whether this notion also holds for HLA mismatches in other loci, as it has been reported that specific combinations of mismatches in HLA-C and HLA-DPB1 may significantly reduce RI and thus improve overall survival rates [28].

\section{The Impact of HLA-DRB1 Mismatches}

HLA class II antigens are heterodimers formed by the products of two genes encoding for an alpha and a beta chain [29]. The HLA-DRB1 gene forms the beta chain of the HLA-DR antigen. It shows the highest diversity among all HLA class II genes. It is also the HLA class II molecule with the highest cell surface expression when compared to other HLA class II antigens [30]. HLA class II is expressed by professional antigen-presenting cells (macrophages, B lymphocytes, and dendritic cells) but may be induced upon activation in other cells [31, 32]. A particularity of HLA-DR is the association with different polymorphic HLA-DRB3, HLA-DRB4, and HLA-DRB5 genes. These form the DR52, DR53, and DR51 antigens, which are however expressed at a lower level [33]. Although the HLA-DRB3/4/5 genes are currently not routinely typed, these associations are important as due to the strong linkage, HLA-DRB1 differences between patients and donors may be linked with additional differences among the HLA-DRB3/4/5 genes. These may contribute to HLA-DRB1 mismatch-associated morbidity particularly if a DR52, DR53, or DR51 antigen is absent in either donor or recipient $[34,35]$. Early studies had indicated that HLA-DRB1 mismatches are particularly risky, and this is the reason why differences at the HLADRB1 locus are often avoided in donor selection [36]. Consequentially, the numbers of patients in large retrospective cohort studies are rather low when compared to HLA-A- or HLA-C-incompatible cases, limiting the power of these analyses. Nevertheless, most studies conducted in Europe or the US showed high risk of mortality and particularly of TRM (Table 2). This high posttransplant morbidity is apparently caused by a high incidence of acute GvHD in these patients, while its effect on chronic GvHD and relapse appears less pronounced. The data from the Japanese Marrow Donor Program are different, showing generally lower hazard ratios for HLA-DRB1 mismatches [37]. Immunogenetic characteristics of the Japanese population that are also reflected in the donor selection process, possibly resulting in identification of donor-recipient combinations with a low immunogenicity, may account for the discrepant outcome results [38]. This is interesting, as the numbers at risk in the Japanese studies are markedly higher when compared to data from Europe and the US. This aspect is further discussed in the section "High Risk and Permissible Allele Combinations."

\section{The Impact of HLA-C Mismatches}

When it was reported that HLA-C differences increased mortality risk in a large National Marrow Donor Program study [39], HLA-C typing and matching was immediately included in many national donor search and matching strategies. This observation has since been repeatedly confirmed. Hazard ratios for the endpoint overall survival ranged between 1.13 and 2.12 [17, 27]. When HLA-C allele and antigen mismatches were analyzed separately, HLA-C antigen differences showed hazard ratios comparable to any other HLA mismatch, while HLA-C allele differences were better tolerated [7, 21]. This is probably caused by the presence of permissible mismatches, which occur quite frequently [40]. In general, the transplantation biology of HLA-C is complex as several factors influence HLA-C-mediated alloreactivity. It has been shown that the expression of HLA-C molecules on the cell surface is variable and depends on the haplotype context as well as on other genetic polymorphisms in the proximity of the HLA-C gene [41, 42]. Indeed, in a clinical study, the inferred HLA-C expression in HLA-C mismatched transplantations correlated with outcome [43]. Additionally, the already mentioned presence of permissible mismatches may confound the analysis of HLA-C allele differences when compared to HLA-C antigen differences. Stratification for permissible mismatches (namely HLA-C ${ }^{*} 03: 03 / 03: 04$ ) may segregate HLA-C allele differences in mismatches with low or no effect on allorecognition and in ones with a detrimental effect on outcome [40]. However, knowledge about permissible mismatch combinations is still incomplete at present, and the clinical evidence to date only supports preferring HLA-C*03:03/03:04 mismatches over other 
HLA differences. Above this, HLA-C molecules act as ligands to KIR receptors. All HLA-C alleles can be separated into two groups. Group 1 is characterized by an asparagine at amino acid position 80, and group 2 is characterized by a lysine at position 80 in the mature protein. HLA-C group 1 alleles interact with the KIR2DL2/3 and KIR2DS2/3 receptors and HLA-C group 2 alleles interact with the KIR2DL1 and KIR2DS1/5 receptors. There is some evidence that these interactions may influence NK-mediated alloreactivity and potentially relapse control, but the evidence is not conclusive at present and is only rarely considered with regard to donor selection [44].

\section{The Impact of HLA-DQB1 Mismatches}

HLA-DQB1 forms the polymorphic beta chain of the HLA-DQ antigen. The HLA-DQA1 gene shows only limited diversity and is currently not routinely typed for HSCT purposes. The HLA-DQ antigen shows markedly lower expression as compared to HLA-DRB1 [45, 46]. Due to the strong linkage within conserved HLA haplotypes, HLA-DQB1 differences are rare if the donor is matched for HLA-A, HLA-B, HLA-C, and particularly HLA-DRB1 [47]. Large retrospective studies could not show a significant effect of HLA-DQB1 matching on HSCT outcome $[7,37]$. This may be due to the low number of DQB1 differences and a resulting low statistical power or lower hazard ratios. The presence of permissible mismatches may also be a possible explanation. In a study with German patients conducted by our group, DQB1 allele differences showed no increased hazard ratios, while HLA-DQB1 antigen differences showed hazard ratios similar to other HLA differences. However, statistical significance was not reached in this subanalysis when corrected for multiple testing [21]. In a recent meta-analysis, HLA-DQB1 differences showed a hazard ratio of 1.07 $(p=0.142)$ for overall survival and 1.30 for TRM ( $p=$ 0.041 ), which was significant, among 3,031 patients at risk and 8,531 controls from three studies [48]. These results suggest that HLA-DQB1 is not completely irrelevant. Further work is needed to define permissible and clinically relevant HLA-DQB1 differences. For this purpose, information about linked HLA-DRB3/4/5 as well as possibly HLA-DQA1 should be included [49]. The available data suggest selection of donors with HLA-DQB1 differences over other HLA differences if no 10/10matched donor is available. However, it should be kept in mind that HLA-DQB1 differences may increase transplant-associated complications as shown in the mentioned meta-analysis and also in a case report, where de novo HLA-DQB1/DQA1 antibodies appeared posttransplant, leading to graft rejection [50].

\section{The Impact of HLA-DPB1 Mismatches}

Early studies have indicated that HLA-DPB1 differences associate with increased GvHD risk, but also with decreased relapse rates $[36,51]$. An impact on mortality was not observed, which is the reason why HLA-DPB1 was not included in typing and matching strategies back then. In a groundbreaking study using in vitro testing based on mixed lymphocyte culture, it was shown that HLA-DPB1 alleles can be grouped according to their immunogenicity into three groups (low, intermediate, high) [52]. This led to the development of TCE-3 grouping, also known as T cell epitope matching according to the combination of donor/recipient alleles. This grouping was later shown to be predictive of HSCT outcome in a large international cohort study from the International Histocompatibility Working Group in Hematopoietic Cell Transplantation [53]. Nonpermissive mismatches were associated with an increased overall mortality (hazard ratio 1.15) and acute GvHD risk (hazard ratio 1.31) when compared to permissive or HLA-DPB1-matched cases. This observation has been repeatedly confirmed since [54-56]. The molecular pattern behind HLA-DPB1 TCE matching has been characterized, allowing grouping of HLA-DPB1 alleles according to functional and structural dissimilarity. The refined TCE-3 algorithm now allows grouping of all HLA-DPB1 alleles [57]. Only polymorphisms within exon 2 seem to determine immunogenicity. Many transplant centers now include HLA-DPB1 T cell epitope matching as an additional criterion in their donor selection strategies if two or more equally suitable donors are available. It must be noted though that due to the low linkage between HLA-DR/DQ and HLA-DP, approximately $80 \%$ of the retrospectively typed donor/recipient pairs are HLA-DPB1-mismatched at one or even two alleles [53]. Furthermore, HLA-DPB1-mediated alloreactivity seems to be influenced by a polymorphism (rs9277534) in the vicinity of the HLA-DPB1 gene. Highly expressed mismatched allotypes led to increased GvHD incidence in one study [58]. It would be interesting to investigate whether a combination of the HLA-DPB1 expression level concept and the TCE-3 algorithm leads to an improved prediction model for HLA-DPB1 matching.

\section{The Mismatch Vector}

The vector of HLA incompatibilities is mostly bidirectional. This means that patient and donor express a different HLA allotype of the mismatched gene. If, however, the patient or the donor is homozygous at the locus concerned, mismatches are incompatible in either the HvG or the $\mathrm{GvH}$ direction. The HvG mismatch implies that the nonshared HLA allotype is only present on donor cells, 
whereas in the GvH incompatibility the nonshared HLA allotype is only present on recipient cells. In one dedicated study, it was observed that transplantations with single mismatches in the GvH vector showed acute GvHD rates similar to those of bidirectional mismatches, whereas mismatches in the HvG vector exhibited acute GvHD rates similar to those of completely matched transplantations. Mortality was, however, not different between the single mismatched groups, which may be due to power issues or an impaired relapse control in the presence of unidirectional HvG mismatches. Nevertheless, the lower GvHD rates suggest that mismatches at a homozygous patient locus may be more preferable than mismatches at a heterozygous locus [59].

\section{High Risk and Permissible Allele Combinations}

The search success rates for 10/10-matched (HLA-A, HLA-B, HLA-C, HLA-DRB1, and HLA-DQB1) donors range from 60 to $80 \%$, depending on the search and transplantation criteria as well as on the patient's HLA haplotype status [60]. For patients with uncommon haplotypes, success rates are poor (as low as $5 \%$ ), even after months of searching [61]. The patient's ethnical background is also of great importance as non-Caucasian ethnicities or individuals with mixed ethnicities are underrepresented in the world donor pool [9]. Therefore, the identification of high-risk and permissive allele combinations is of importance if no matched donor is available. Many approaches have been undertaken in order to identify such combinations, including in silico methods like the Predicted Indirectly Recognizable HLA Epitopes (PIRCHE), which determines potentially immunogenic peptides deriving from mismatched HLA alleles presented by the shared HLA alleles, and the online tool HistoCheck, which determines a dissimilarity score between mismatched HLA alleles. The HistoCheck score did not show any predictive value, but there is some evidence suggesting that higher PIRCHE numbers associate with adverse outcome in unrelated stem cell transplantation $[62,63]$. The biological and functional roles of AA substitutions in key positions of the HLA molecule have been demonstrated to be responsible for severe acute GvHD [64, 65]. All of the identified crucial AA substitutions are either located in the peptide-binding pockets (beta-plated sheet positions 9, 99, and 116; alpha helix of HLA class I position 156) or at killer immunoglobulin-like receptor binding positions (HLA class I positions 77 and 80). Strikingly, these results suggest that two of the most common HLA-C mismatches in recipient-donor pairs, $C^{*} 15: 02$ 14:02 and $C^{*} 04: 01-12: 03$, should be considered as highrisk mismatches since they differ by 5-6 key AA substations [66]. The Japanese Marrow Donor Program re- search group identified several high-risk allele mismatch combinations, e.g., HLA-A*02:01-02:06, HLA-A*26:0226:01, HLA-B*15:01-15:07, and HLA-C combinations where either $C^{*} 03: 03$ or $C^{*} 03: 04$ had been mismatched with $C^{*} 04: 01, C^{*} 08: 01, C^{*} 14: 02$, or $C^{*} 15: 02$ [66]. For HLA class II they identified HLA-DRB $1^{*} 04: 05-04: 03$, HLA-DPB1*03:01-05:01, and DPB1*05:01-09:01 as being high-risk mismatches. However, only the HLA-A and HLA-C mismatch combinations could be linked to the subsequently identified AA substitutions (B9, C99, and C116). Hence, identification and avoidance of the aforementioned AA mismatches alone does not guarantee a better outcome after mismatched HSCT. In another study the mismatch combinations HLA-DRB1*11:01-11:04 and HLA-DQB $1{ }^{*}$ 03:01-03:02 were proposed as low-risk HLA differences [17]. One of the first allele mismatches that was considered to be permissible was $C^{*} 03: 03-03: 04$, as indicated by cytotoxic $\mathrm{T}$ cell precursor frequency assays [67] and later on supported by a large-scale study showing that single mismatched $C^{*} 03: 03-03: 04$-transplanted patients had a similar outcome compared to 8/8-matched patients [40]. These two alleles differ in only one AA. In contrast, the HLA-B*44:02-44:03 allele mismatch (also only one AA difference) has been shown to induce powerful alloreactivity [68]. In summary, the current evidence suggests selection of single HLA-C allele (particularly the combination HLA-C*03:03/03:04) or single HLA-DQB1 mismatches over donors with other HLA differences.

\section{HLA Matching in CB Transplantation}

The role of HLA matching in umbilical CB HSCT has been controversially discussed for almost two decades. Whereas some research groups reported a beneficial influence of HLA matching, other publications demonstrated positive effects of HLA-mismatched grafts. As a result, the National Marrow Donor Program as well as the Blood and Marrow Transplant Clinical Trials Network both only recommend the use of high- or intermediateresolution HLA-A, HLA-B, and high-resolution HLADRB1 typed and at least 4/6 HLA-matched CB units [10, 69]. In a study comparing transplantation outcomes of pediatric patients with hematologic malignancies transplanted with high-resolution HLA-matched and -mismatched grafts, the incidence of severe (grade III-IV) GvHD was significantly increased if the transplant pair was mismatched for more than one HLA allele [70]. However, these results are limited by small case numbers, especially regarding 6/6-matched transplantations (16 out of 179), and do not allow definitive conclusions. In adults, data on the impact of HLA matching on CB HSCT are more conclusive. Regarding the overall effect of HLA dis- 
parity, the risk of TRM was increased if a single or two or more HLA mismatches were present [71]. A large-scale study that included 1,202 CB transplantations facilitated by the New York Blood Center National Cord Blood Program demonstrated striking differences between subgroups of patients with unidirectional mismatches. Transplantations with mismatches in the $\mathrm{GvH}$ direction only $(\mathrm{GvH}-\mathrm{O})$ did not show lower TRM or treatment failure rates compared to 6/6-matched CB transplantations and exhibited comparable neutrophil and platelet engraftment rates [72]. Mismatches in the HvG rejection direction only, however, were detrimental as such mismatched transplantations showed significantly delayed engraftment and increased risk of relapse in this patient group when compared to the GvH-O group. A particular group of possibly permissive HLA mismatches consists of the so-called noninherited maternal antigens (NIMAs). During pregnancy, the developing fetus is exposed to maternal cells expressing noninherited HLA antigens and thereby develops tolerance to these HLA-mismatched NIMAs [73]. It was shown that NIMA-matched CB transplantations show similar outcomes to 6/6-matched transplantations $[74,75]$. Compared to NIMA-mismatched transplantations, TRM and treatment failure rates were lower.

\section{HLA Antibodies}

HLA alloimmunization occurs mostly upon exposure to HLA antigens during pregnancy, transfusion, or transplantation [76]. In the transplant setting, preformed antiHLA antibodies in patients or donors may exist. Most relevant are preformed donor-specific HLA antibodies in patients. Prevalence of such antibodies has been associated with a high risk of graft failure in unrelated stem cell transplantation [77]. The prevalence of HLA alloimmunization in patients undergoing HSCT ranges between 19.6 and $39.4 \%$, and the prevalence of donor-specific alloimmunization depends strongly on the donor source, with rates up to $83 \%$ in haploidentical transplantation or CB transplantation due to the larger number of HLA mismatches as compared to matched unrelated or matched related transplantation [78]. Therefore, patient antibody screening is recommended routinely in these instances [79]. Particularly complement fixing antibodies, as detected by the $\mathrm{C} 1 \mathrm{q}$ assay in Luminex SAB testing, has been reported as highly predictive of graft failure [80]. Higher levels of antibodies as determined by median fluorescence intensity seem also to associate with higher rates of graft failure. Reduction of pretransplant HLA antibodies has been attempted with rituximab in combination with plasmapheresis/immunoadsorption or by infusion of corresponding antigen-bearing platelets, but often only with limited success. Donor-preformed HLA antibodies may lead to passenger lymphocyte syndrome, characterized by induction of high antibody levels 7-10 days after transplantation, which gradually decline afterwards and disappear within 3 months [81]. A higher risk for occurrence of passenger lymphocyte syndrome has been reported in HLA-incompatible transplantations and with peripheral blood stem cells as graft source. Clinical consequences may be a decrease in thrombocyte counts and thrombocyte transfusion refractoriness. Theoretically, the incidence and severity of acute GvHD may be influenced; however, data on this issue are sparse [82]. De novo HLA antibodies after transplantation are a common problem in solid organ transplantation, but occur only seldom in HSCT. Nevertheless, they may lead to graft failure in some cases [83]. In chronic GvHD, antibody-mediated processes have been postulated to play a significant role, particularly in the context of skin affection [84]. Whether HLA antibodies play a role in this situation has not been conclusively evaluated yet. The presence of donor-specific HLA antibodies has not been comprehensively assessed in most of the major studies and may contribute to the detrimental effect of HLA mismatches to some smaller extent, as the vast majority of patients do not exhibit donor-specific, high-titer, $\mathrm{Clq}+$ HLA antibodies.

\section{Summary and Perspective}

In unrelated donor selection, matching for the classical high-expression loci (HLA-A, HLA-B, HLA-C, HLADRB1) is still the paramount criterion. Also important factors to consider are CMV compatibility and donor age. If more than one equally suitable donors are available, further parameters such as DPB1 epitope matching, blood group, and donor sex may be considered. With regard to HLA typing, the introduction of benchtop sequencers and targeted sequencing kits for HLA genes marks another quantum leap in the evolution of HLA diagnostics. The current industry standard for these kits is complete or nearly complete gene typing for HLA-A, HLA-B, HLA-C, HLA-DRB1, HLA-DRB3/4/5, HLADQA1, HLA-DQB1, HLA-DPA1, and HLA-DPB1 using amplification with long-range PCR and shotgun sequencing with short read technologies. This approach offers several benefits in reliability and depth of resolution. Besides the reduction of ambiguities, these assays allow for detection of most or all nonexpressed alleles. These socalled null alleles may lead to a missing antigen in either donor or recipient and may remain undetected in conventional typing methods if the causing polymorphism is outside of the amplified region (usually the antigen recognition domain, ARC). Such differences are highly clin- 
Table 3. The frequency of HLA null alleles including HLADRB3/4/5 genes

\begin{tabular}{lll}
\hline & Allele & Frequency \\
\hline 1 & DRB4*01:03:01:02N & 0.03572 \\
2 & C $^{*} 04: 09 \mathrm{~N}$ & $0.00052-0.00142$ \\
3 & DRB4*03:01N & 0.00019 \\
4 & DRB5*01:08N & 0.00013 \\
5 & $\mathrm{~B}^{*} 51: 11 \mathrm{~N}$ & $0.00011-0.00019$ \\
6 & $\mathrm{~A}^{*} 24: 09 \mathrm{~N}$ & 0.00012 \\
7 & $\mathrm{~A}^{*} 01: 04 \mathrm{~N}$ & 0.00011 \\
\hline
\end{tabular}

ically relevant as they must be viewed as full antigen mismatch. Table 3 shows the most frequent nonexpressed HLA alleles including HLA-DRB3/4/5 based on our registry donor typing and data from the Zentrales Knochenmarkspender-Register Deutschland [85]. Another benefit is the inclusion of additional genes (HLA-DRB3/4/5, HLA-DQA1, and HLA-DPA1). These may enable identification of unusual haplotype associations, which may also be associated with the presence of absence of an antigen $[86,87]$. Furthermore, differences within the ARC of these genes may be relevant in some situations as epitopes are formed by the alpha and the beta chain together. This may enable generation of donor-specific antibodies even if the beta chain is matched between donor and recipient, possibly leading to graft rejection [50]. One study has already shown that the cumulative number of differences for the low-expression loci HLA-DRB3/4/5, HLADQ, and HLA-DP associate with adverse outcome [49]. It is likely that future large cohort studies will show that inclusion of these loci in typing and matching strategies for unrelated donor search is beneficial for HSCT outcome. Moreover, the definition of coding polymorphisms outside the ARC is possible with these tests, sparking intense research evaluating their impact on unrelated HSCT. In a study conducted by the Anthony Nolan Research Institute, $29.1 \%$ of transplant pairs showed differences outside the ARC in transplant pairs thought to be 12/12-matched based on conventional typing. Fulllength-matched transplant pairs had a better survival as compared to ARC-matched but full-length-mismatched transplant pairs including intronic and UTR variations [88]. Novel matching approaches for full-length HLA sequences may identify supermatched donor/recipient combinations leading to lower GvHD and better survival [89]. Another interesting concept is the evaluation of haplotype matching in unrelated stem cell transplantation, i.e., the matching of conserved extended HLA haplotypes, which may improve survival due to matching of potential relevant polymorphisms beyond the typed antigens $[90,91]$. Lastly, the high throughput of next-generation sequencing technology allows inclusion of further parameters, for which preliminary data show relevance in unrelated HSCT, like HLA-E and MICA [92-94]. In summary, the novel next-generation sequencing completegene assays improve histocompatibility testing for patients and donors in HSCT and will likely lead to a further improvement of HSCT outcomes in the future.

\section{Disclosure Statement}

The authors declare no conflicts of interest.

\section{References}

1 Dausset J. Iso-leuko-antibodies. Acta Haematol. 1958 Jul-Oct;20(1-4):156-66. French.

2 Doherty PC, Zinkernagel RM. A biological role for the major histocompatibility antigens. Lancet. 1975 Jun;1(7922):1406-9.

3 Negrin RS. Graft-versus-host disease versus graft-versus-leukemia. Hematology (Am Soc Hematol Educ Program). 2015;2015(1):22530 .

4 Zeiser R, Blazar BR. Acute Graft-versus-Host Disease - Biologic Process, Prevention, and Therapy. N Engl J Med. 2017 Nov;377(22): 2167-79.

5 Passweg JR, Baldomero H, Bader P, Basak GW, Bonini C, Duarte R, et al.; European Society for Blood and Marrow Transplantation (EBMT). Is the use of unrelated donor transplantation leveling off in Europe? The 2016 European Society for Blood and Marrow Transplant activity survey report. Bone Marrow Transplant. 2018 Sep;53(9):1139-48.

6 Sureda A, Bader P, Cesaro S, Dreger P, Duarte $\mathrm{RF}$, Dufour C, et al. Indications for allo- and auto-SCT for haematological diseases, solid tumours and immune disorders: current practice in Europe, 2015. Bone Marrow Transplant. 2015 Aug;50(8):1037-56.

7 Lee SJ, Klein J, Haagenson M, Baxter-Lowe LA, Confer DL, Eapen M, et al. High-resolution donor-recipient HLA matching contributes to the success of unrelated donor marrow transplantation. Blood. 2007 Dec;110(13):4576-83.

8 Sengsayadeth S, Savani BN, Blaise D, Malard F, Nagler A, Mohty M. Reduced intensity conditioning allogeneic hematopoietic cell transplantation for adult acute myeloid leukemia in complete remission - a review from the Acute Leukemia Working Party of the EBMT. Haematologica. 2015 Jul;100(7):859-69.

9 Gragert L, Eapen M, Williams E, Freeman J, Spellman S, Baitty R, et al. HLA match likelihoods for hematopoietic stem-cell grafts in the U.S. registry. N Engl J Med. 2014 Jul; 371(4):339-48.

10 Howard CA, Fernandez-Vina MA, Appelbaum FR, Confer DL, Devine SM, Horowitz $\mathrm{MM}$, et al. Recommendations for donor human leukocyte antigen assessment and matching for allogeneic stem cell transplantation: consensus opinion of the Blood and
Marrow Transplant Clinical Trials Network (BMT CTN). Biol Blood Marrow Transplant. 2015 Jan;21(1):4-7.

11 Scott I, O’Shea J, Bunce M, Tiercy JM, Argüello JR, Firman H, et al. Molecular typing shows a high level of HLA class I incompatibility in serologically well matched donor/patient pairs: implications for unrelated bone marrow donor selection. Blood. $1998 \mathrm{Dec}$;92(12): 4864-71.

12 Tiercy JM. How to select the best available related or unrelated donor of hematopoietic stem cells? Haematologica. 2016 Jun;101(6): 680-7.

13 Robinson J, Halliwell JA, Hayhurst JD, Flicek P, Parham P, Marsh SG. The IPD and IMGT/ HLA database: allele variant databases. $\mathrm{Nu}-$ cleic Acids Res. 2015 Jan;43(Database issue):D423-31.

14 Yanover C, Petersdorf EW, Malkki M, Gooley T, Spellman S, Velardi A, et al. HLA mismatches and hematopoietic cell transplantation: structural simulations assess the impact of changes in peptide binding specificity on transplant outcome. Immunome Res. 2011;7(2):4. 
15 Iacobelli S; EBMT Statistical Committee. Suggestions on the use of statistical methodologies in studies of the European Group for Blood and Marrow Transplantation. Bone Marrow Transplant. 2013 Mar;48(Suppl 1): S1-37.

16 Apps R, Meng Z, Del Prete GQ, Lifson JD, Zhou M, Carrington M. Relative expression levels of the HLA class-I proteins in normal and HIV-infected cells. J Immunol. $2015 \mathrm{Apr}$; 194(8):3594-600

17 Passweg JR, Schanz U, Chalandon Y, Güngör T, Baldomero H, Heim D, et al.; Swiss Blood Stem Cell Transplantation Group. High-resolution HLA matching in unrelated donor transplantation in Switzerland: differential impact of class I and class II mismatches may reflect selection of nonimmunogenic or weakly immunogenic DRB1/DQB1 disparities. Bone Marrow Transplant. 2015 Sep; 50(9):1201-5

18 Woolfrey A, Klein JP, Haagenson M, Spellman S, Petersdorf E, Oudshoorn M, et al. HLA-C antigen mismatch is associated with worse outcome in unrelated donor peripheral blood stem cell transplantation. Biol Blood Marrow Transplant. 2011 Jun;17(6): 885-92.

19 Kanda Y, Kanda J, Atsuta Y, Maeda Y, Ichinohe T, Ohashi K, et al. Impact of a single human leucocyte antigen (HLA) allele mismatch on the outcome of unrelated bone marrow transplantation over two time periods. A retrospective analysis of 3003 patients from the HLA Working Group of the Japan Society for Blood and Marrow Transplantation. Br J Haematol. 2013 May;161(4):566-77.

20 Ayuk F, Beelen DW, Bornhäuser M, Stelljes M, Zabelina T, Finke J, et al. Relative impact of HLA matching and non-HLA donor characteristics on outcomes of allogeneic stem cell transplantation for acute myeloid leukemia and myelodysplastic syndrome. Biol Blood Marrow Transplant. 2018 Dec;24(12):255867.

21 Fürst D, Müller C, Vucinic V, Bunjes D, Herr W, Gramatzki M, et al. High-resolution HLA matching in hematopoietic stem cell transplantation: a retrospective collaborative analysis. Blood. 2013 Oct;122(18):3220-9.

22 Yokoyama H, Kanda J, Fuji S, Kim SW, Fukuda T, Najima Y, et al.; HLA Working Group of the Japan Society for Hematopoietic Cell Transplantation. Impact of Human Leukocyte Antigen Allele Mismatch in Unrelated Bone Marrow Transplantation with Reduced-Intensity Conditioning Regimen. Biol Blood Marrow Transplant. 2017 Feb;23(2): 300-9.

23 Distler E, Bloetz A, Albrecht J, Asdufan S, Hohberger A, Frey M, et al. Alloreactive and leukemia-reactive $\mathrm{T}$ cells are preferentially derived from naive precursors in healthy donors: implications for immunotherapy with memory T cells. Haematologica. 2011 Jul; 96(7):1024-32.

24 Marie-Cardine A, Divay F, Dutot I, Green A, Perdrix A, Boyer O, et al. Transitional B cells in humans: characterization and insight from B lymphocyte reconstitution after hematopoietic stem cell transplantation. Clin Immunol. 2008 Apr;127(1):14-25.
25 Nunes E, Heslop H, Fernandez-Vina M, Taves C, Wagenknecht DR, Eisenbrey AB, et al.; Harmonization of Histocompatibility Typing Terms Working Group. Definitions of histocompatibility typing terms: Harmonization of Histocompatibility Typing Terms Working Group. Hum Immunol. 2011 Dec; 72(12):1214-6.

26 Flowers ME, Inamoto Y, Carpenter PA, Lee SJ, Kiem HP, Petersdorf EW, et al. Comparative analysis of risk factors for acute graft-versus-host disease and for chronic graft-versushost disease according to National Institutes of Health consensus criteria. Blood. 2011 Mar;117(11):3214-9.

27 Verneris MR, Lee SJ, Ahn KW, Wang HL, Battiwalla M, Inamoto Y, et al. HLA Mismatch Is Associated with Worse Outcomes after Unrelated Donor Reduced-Intensity Conditioning Hematopoietic Cell Transplantation: An Analysis from the Center for International Blood and Marrow Transplant Research. Biol Blood Marrow Transplant. 2015 Oct;21(10):1783-9.

28 Kawase T, Matsuo K, Kashiwase K, Inoko H, Saji H, Ogawa S, et al.; Japan Marrow Donor Program. HLA mismatch combinations associated with decreased risk of relapse: implications for the molecular mechanism. Blood. 2009 Mar;113(12):2851-8.

29 Stern LJ, Brown JH, Jardetzky TS, Gorga JC, Urban RG, Strominger JL, et al. Crystal structure of the human class II MHC protein HLADR1 complexed with an influenza virus peptide. Nature. 1994 Mar;368(6468):215-21.

30 Brooks CF, Moore M. Differential MHC class II expression on human peripheral blood monocytes and dendritic cells. Immunology. 1988 Feb;63(2):303-11.

31 Reith W, LeibundGut-Landmann S, Waldburger JM. Regulation of MHC class II gene expression by the class II transactivator. Nat Rev Immunol. 2005 Oct;5(10):793-806.

32 Chang $\mathrm{CH}$, Hong SC, Hughes CC, Janeway CA Jr, Flavell RA. CIITA activates the expression of MHC class II genes in mouse T cells. Int Immunol. 1995 Sep;7(9):1515-8.

33 Emery P, Mach B, Reith W. The different level of expression of HLA-DRB1 and -DRB3 genes is controlled by conserved isotypic differences in promoter sequence. Hum Immunol. 1993 Oct;38(2):137-47.

34 Detrait M, Morisset S, Chalandon Y, YakoubAgha I, Dufossé F, Labalette M, et al. Suggestive evidence of a role of HLA-DRB4 mismatches in the outcome of allogeneic hematopoietic stem cell transplantation with HLA-10/10-matched unrelated donors: a French-Swiss retrospective study. Bone Marrow Transplant. 2015 Oct;50(10):1316-20.

35 Grubic Z, Maskalan M, Radmanic L, Stingl Jankovic K, Burek Kamenaric M, Zunec R. The distribution of the DRB4*01:03:01:02N null allele in HLA-DRB1 DQB1 haplotypes in the Croatian population. HLA. 2018 Jan; 91(1):23-8.

36 Petersdorf EW, Longton GM, Anasetti C, Martin PJ, Mickelson EM, Smith AG, et al. The significance of HLA-DRB1 matching on clinical outcome after HLA-A, B, DR identical unrelated donor marrow transplantation. Blood. 1995 Aug;86(4):1606-13.
37 Morishima Y, Kashiwase K, Matsuo K, Azuma F, Morishima S, Onizuka M, et al.; Japan Marrow Donor Program. Biological significance of HLA locus matching in unrelated donor bone marrow transplantation. Blood. 2015 Feb;125(7):1189-97.

38 Saito S, Ota S, Yamada E, Inoko H, Ota M. Allele frequencies and haplotypic associations defined by allelic DNA typing at HLA class I and class II loci in the Japanese population. Tissue Antigens. 2000 Dec;56(6):522-9.

39 Flomenberg N, Baxter-Lowe LA, Confer D, Fernandez-Vina M, Filipovich A, Horowitz $\mathrm{M}$, et al. Impact of HLA class I and class II high-resolution matching on outcomes of unrelated donor bone marrow transplantation: HLA-C mismatching is associated with a strong adverse effect on transplantation outcome. Blood. 2004 Oct;104(7):1923-30.

40 Fernandez-Viña MA, Wang T, Lee SJ, Haagenson M, Aljurf M, Askar M, et al. Identification of a permissible HLA mismatch in hematopoietic stem cell transplantation. Blood. 2014 Feb;123(8):1270-8.

41 Apps R, Qi Y, Carlson JM, Chen H, Gao X, Thomas R, et al. Influence of HLA-C expression level on HIV control. Science. 2013 Apr; 340(6128):87-91

42 Thomas R, Apps R, Qi Y, Gao X, Male V, O'hUigin C, et al. HLA-C cell surface expression and control of HIV/AIDS correlate with a variant upstream of HLA-C. Nat Genet. 2009 Dec;41(12):1290-4.

43 Petersdorf EW, Gooley TA, Malkki M, Bacigalupo AP, Cesbron A, Du Toit E, et al.; International Histocompatibility Working Group in Hematopoietic Cell Transplantation. HLA-C expression levels define permissible mismatches in hematopoietic cell transplantation. Blood. 2014 Dec;124(26):3996-4003.

44 Venstrom JM, Pittari G, Gooley TA, Chewning $\mathrm{JH}$, Spellman $\mathrm{S}$, Haagenson $\mathrm{M}$, et al. HLA-C-dependent prevention of leukemia relapse by donor activating KIR2DS1. N Engl J Med. 2012 Aug;367(9):805-16.

45 Nuñez G, Ball EJ, Myers LK, Stastny P. Allostimulating cells in man. Quantitative variation in the expression of HLA-DR and HLADQ molecules influences T-cell activation. Immunogenetics. 1985;22(1):85-91.

46 Cesari M, Caillens H, Cadet F, Pabion M. In vivo analysis of HLA-DQ gene expression in heterozygous cell lines. Immunogenetics. 1999 Dec;50(5-6):309-18.

47 Sanchez-Mazas A, Djoulah S, Busson M, Le Monnier de Gouville I, Poirier JC, Dehay C, et al. A linkage disequilibrium map of the MHC region based on the analysis of 14 loci haplotypes in 50 French families. Eur J Hum Genet. 2000 Jan;8(1):33-41.

48 Tie R, Zhang T, Yang B, Fu H, Han B, Yu J, et al. Clinical implications of HLA locus mismatching in unrelated donor hematopoietic cell transplantation: a meta-analysis. Oncotarget. 2017 Apr;8(16):27645-60.

49 Fernández-Viña MA, Klein JP, Haagenson M, Spellman SR, Anasetti C, Noreen H, et al. Multiple mismatches at the low expression HLA loci DP, DQ, and DRB3/4/5 associate with adverse outcomes in hematopoietic stem cell transplantation. Blood. 2013 May; 121(22):4603-10. 
50 Yabe H, Morimoto T, Takakura H, Okuya M, Ikegaya R, Kato S, et al. Post-transplantationemerging anti-HLA DQA1/DQB1 antibody possibly responsible for graft rejection after myeloablative-unrelated marrow grafting. Bone Marrow Transplant. 2016 Apr;51(4): 601-3.

51 Shaw BE, Marsh SG, Mayor NP, Russell NH, Madrigal JA. HLA-DPB1 matching status has significant implications for recipients of unrelated donor stem cell transplants. Blood. 2006 Feb;107(3):1220-6.

52 Zino E, Frumento G, Marktel S, Sormani MP, Ficara F, Di Terlizzi S, et al. A T-cell epitope encoded by a subset of HLA-DPB1 alleles determines nonpermissive mismatches for hematologic stem cell transplantation. Blood. 2004 Feb;103(4):1417-24.

53 Fleischhauer K, Shaw BE, Gooley T, Malkki M, Bardy P, Bignon JD, et al.; International Histocompatibility Working Group in $\mathrm{He}$ matopoietic Cell Transplantation. Effect of T-cell-epitope matching at HLA-DPB1 in recipients of unrelated-donor haemopoietic-cell transplantation: a retrospective study. Lancet Oncol. 2012 Apr;13(4):36674.

54 Pidala J, Lee SJ, Ahn KW, Spellman S, Wang HL, Aljurf M, et al. Nonpermissive HLADPB1 mismatch increases mortality after myeloablative unrelated allogeneic hematopoietic cell transplantation. Blood. 2014 Oct; 124(16):2596-606.

55 Burek Kamenaric M, Maskalan M, Grubic Z, Mikulic M, Serventi Seiwerth R, Durakovic N, et al. HLA-DPB1 matching in unrelated hematopoietic stem cell transplantation program contributes to a higher incidence of disease relapse. Hum Immunol. 2017 Nov; 78(11-12):665-71.

56 Moyer AM, Hashmi SK, Kroning CM, Kremers WK, De Goey SR, Patnaik M, et al. Clinical outcomes of HLA-DPB1 mismatches in 10/10 HLA-matched unrelated donor-recipient pairs undergoing allogeneic stem cell transplant. Eur J Haematol. 2017 Sep;99(3): 275-82.

57 Crivello P, Zito L, Sizzano F, Zino E, Maiers M, Mulder A, et al. The impact of amino acid variability on alloreactivity defines a functional distance predictive of permissive HLA-DPB1 mismatches in hematopoietic stem cell transplantation. Biol Blood Marrow Transplant. 2015 Feb;21(2):23341.

58 Petersdorf EW, Malkki M, O’hUigin C, Carrington $\mathrm{M}$, Gooley $\mathrm{T}$, Haagenson $\mathrm{MD}$, et al. High HLA-DP Expression and Graft-versusHost Disease. N Engl J Med. 2015 Aug;373(7): 599-609.

59 Hurley CK, Woolfrey A, Wang T, Haagenson M, Umejiego J, Aljurf M, et al. The impact of HLA unidirectional mismatches on the outcome of myeloablative hematopoietic stem cell transplantation with unrelated donors. Blood. 2013 Jun;121(23):4800-6.

60 Tiercy JM, Nicoloso G, Passweg J, Schanz U, Seger R, Chalandon Y, et al. The probability of identifying a 10/10 HLA allele-matched unrelated donor is highly predictable. Bone Marrow Transplant. 2007 Sep;40(6):51522.
61 Hirv K, Bloch K, Fischer M, Einsiedler B, Schrezenmeier H, Mytilineos J. Prediction of duration and success rate of unrelated hematopoietic stem cell donor searches based on the patient's HLA-DRB1 allele and DRB1DQB1 haplotype frequencies. Bone Marrow Transplant. 2009 Oct;44(7):433-40.

62 Spellman S, Klein J, Haagenson M, Askar M, Baxter-Lowe LA, He J, et al. Scoring HLA class I mismatches by HistoCheck does not predict clinical outcome in unrelated hematopoietic stem cell transplantation. Biol Blood Marrow Transplant. 2012 May;18(5):739-46.

63 Geneugelijk K, Thus KA, van Deutekom HW, Calis JJ, Borst E, Keșmir C, et al. Exploratory Study of Predicted Indirectly ReCognizable HLA Epitopes in Mismatched Hematopoietic Cell Transplantations. Front Immunol. 2019 Apr; 10:880.

64 Ferrara GB, Bacigalupo A, Lamparelli T, Lanino E, Delfino L, Morabito A, et al. Bone marrow transplantation from unrelated donors: the impact of mismatches with substitutions at position 116 of the human leukocyte antigen class I heavy chain. Blood. 2001 Nov; 98(10):3150-5.

65 Pidala J, Wang T, Haagenson M, Spellman SR, Askar M, Battiwalla M, et al. Amino acid substitution at peptide-binding pockets of HLA class I molecules increases risk of severe acute GVHD and mortality. Blood. 2013 Nov; 122(22):3651-8.

66 Kawase T, Morishima Y, Matsuo K, Kashiwase K, Inoko H, Saji H, et al.; Japan Marrow Donor Program. High-risk HLA allele mismatch combinations responsible for severe acute graft-versus-host disease and implication for its molecular mechanism. Blood. 2007 Oct;110(7):2235-41.

67 Oudshoorn M, Doxiadis II, van den BergLoonen PM, Voorter CE, Verduyn W, Claas $\mathrm{FH}$. Functional versus structural matching: can the CTLp test be replaced by HLA allele typing? Hum Immunol. 2002 Mar;63(3):17684.

68 Macdonald WA, Purcell AW, Mifsud NA, Ely LK, Williams DS, Chang L, et al. A naturally selected dimorphism within the HLA-B44 supertype alters class I structure, peptide repertoire, and T cell recognition. J Exp Med. 2003 Sep;198(5):679-91.

69 Spellman SR, Eapen M, Logan BR, Mueller C, Rubinstein P, Setterholm MI, et al.; National Marrow Donor Program; Center for International Blood and Marrow Transplant Research. A perspective on the selection of unrelated donors and cord blood units for transplantation. Blood. 2012 Jul;120(2):259-65.

70 Kurtzberg J, Prasad VK, Carter SL, Wagner JE, Baxter-Lowe LA, Wall D, et al.; COBLT Steering Committee. Results of the Cord Blood Transplantation Study (COBLT): clinical outcomes of unrelated donor umbilical cord blood transplantation in pediatric patients with hematologic malignancies. Blood. 2008 Nov; 112(10):4318-27.

71 Eapen M, Klein JP, Sanz GF, Spellman S, Ruggeri A, Anasetti C, et al.; Eurocord-European Group for Blood and Marrow Transplantation; Netcord; Center for International Blood and Marrow Transplant Research. Effect of donor-recipient HLA matching at HLA A, B,
C, and DRB1 on outcomes after umbilicalcord blood transplantation for leukaemia and myelodysplastic syndrome: a retrospective analysis. Lancet Oncol. 2011 Dec;12(13): 1214-21.

72 Stevens CE, Carrier C, Carpenter C, Sung D, Scaradavou A. HLA mismatch direction in cord blood transplantation: impact on outcome and implications for cord blood unit selection. Blood. 2011 Oct;118(14):3969-78.

73 Bracamonte-Baran W, Burlingham W. Noninherited maternal antigens, pregnancy, and allotolerance. Biomed J. 2015 Jan-Feb;38(1): 39-51.

74 Rocha V, Spellman S, Zhang MJ, Ruggeri A Purtill D, Brady C, et al.; Eurocord-European Blood and Marrow Transplant Group and the Center for International Blood and Marrow Transplant Research. Effect of HLA-matching recipients to donor noninherited maternal antigens on outcomes after mismatched umbilical cord blood transplantation for hematologic malignancy. Biol Blood Marrow Transplant. 2012 Dec;18(12):1890-6.

75 van Rood JJ, Stevens CE, Smits J, Carrier C, Carpenter C, Scaradavou A. Reexposure of cord blood to noninherited maternal HLA antigens improves transplant outcome in hematological malignancies. Proc Natl Acad Sci USA. 2009 Nov;106(47):19952-7.

76 Kakaiya RM, Triulzi DJ, Wright DJ, Steele WR, Kleinman SH, Busch MP, et al.; National Heart, Lung, and Blood Institute (NHLBI) Retrovirus Epidemiology Donor Study-II. Prevalence of HLA antibodies in remotely transfused or alloexposed volunteer blood donors. Transfusion. 2010 Jun;50(6):132834.

77 Spellman S, Bray R, Rosen-Bronson S, Haagenson $\mathrm{M}$, Klein J, Flesch $\mathrm{S}$, et al. The detection of donor-directed, HLA-specific alloantibodies in recipients of unrelated hematopoietic cell transplantation is predictive of graft failure. Blood. 2010 Apr;115(13):27048.

78 Morin-Zorman S, Loiseau P, Taupin JL, Caillat-Zucman S. Donor-Specific Anti-HLA Antibodies in Allogeneic Hematopoietic Stem Cell Transplantation. Front Immunol. 2016 Aug; 7:307.

79 Ciurea SO, Cao K, Fernandez-Vina M, Kongtim P, Malki MA, Fuchs E, et al. The European Society for Blood and Marrow Transplantation (EBMT) Consensus Guidelines for the Detection and Treatment of Donor-specific Anti-HLA Antibodies (DSA) in Haploidentical Hematopoietic Cell Transplantation. Bone Marrow Transplant. 2018 May; 53(5):521-34

80 Ciurea SO, Thall PF, Milton DR, Barnes TH, Kongtim P, Carmazzi Y, et al. ComplementBinding Donor-Specific Anti-HLA Antibodies and Risk of Primary Graft Failure in Hematopoietic Stem Cell Transplantation. Biol Blood Marrow Transplant. 2015 Aug;21(8): 1392-8.

81 Taniguchi K, Yoshihara S, Maruya E, Ikegame K, Kaida K, Hayashi K, et al. Donor-derived HLA antibody production in patients undergoing SCT from HLA antibody-positive donors. Bone Marrow Transplant. 2012 Oct; 47(10):1338-42. 
82 Delbos F, Barhoumi W, Cabanne L, Beckerich F, Robin C, Redjoul R, et al. Donor Immunization Against Human Leukocyte Class II Antigens is a Risk Factor for Graft-versusHost Disease. Biol Blood Marrow Transplant. 2016 Feb;22(2):292-9.

83 Hefazi M, Hogan WJ, Wakefield LL, Gandhi MJ. The association of de novo anti-HLADPB1 donor-specific antibody formation and primary graft failure after allogeneic hematopoietic cell transplantation. Hum Immunol. 2018 Dec;79(12):861-4.

84 Jin H, Ni X, Deng R, Song Q, Young J, Cassady $\mathrm{K}$, et al. Antibodies from donor B cells perpetuate cutaneous chronic graft-versus-host disease in mice. Blood. 2016 May;127(18): 2249-60.

85 Eberhard HP, Feldmann U, Bochtler W, Baier D, Rutt C, Schmidt AH, et al. Estimating unbiased haplotype frequencies from stem cell donor samples typed at heterogeneous resolutions: a practical study based on over 1 million German donors. Tissue Antigens. 2010 Nov; 76(5):352-61.
86 Nesci S, Talevi N, Andreani M, Manna M, Iliescu A, Lucarelli G. An unusual DRB1*1503 haplotype without a detectable DRB5 locus in a black African family. Tissue Antigens. 1997 Jan;49(1):53-5.

87 Perrier P, Leotard B, Henry MA. A novel HLA haplotype containing a DRB5 gene associated with the DRB ${ }^{*} 0103$ allele. Tissue Antigens. 1993 Nov;42(5):518-21.

88 Mayor NP, Hayhurst JD, Turner TR, Szydlo RM, Shaw BE, Bultitude WP, et al. Recipients Receiving Better HLA-Matched Hematopoietic Cell Transplantation Grafts, Uncovered by a Novel HLA Typing Method, Have Superior Survival: A Retrospective Study. Biol Blood Marrow Transplant. 2019 Mar;25(3):443-50.

89 Vazirabad I, Chhabra S, Nytes J, Mehra V, Narra RK, Szabo A, et al. Direct HLA Genetic Comparisons Identify Highly Matched Unrelated Donor-Recipient Pairs with Improved Transplantation Outcome. Biol Blood Marrow Transplant. 2019 May;25(5):921-31.

90 Jöris MM, Lankester AC, von dem Borne PA, Kuball J, Bierings M, Cornelissen JJ, et al. The impact of frequent HLA haplotypes in high linkage disequilibrium on donor search and clinical outcome after unrelated haematopoietic SCT. Bone Marrow Transplant. 2013 Apr; 48(4):483-90.
91 PetersdorfEW, Malkki M, Gooley TA, Martin PJ, Guo Z. MHC haplotype matching for unrelated hematopoietic cell transplantation. PLoS Med. 2007 Jan;4(1):e8.

92 Carapito R, Jung N, Kwemou M, Untrau M, Michel S, Pichot A, et al. Matching for the nonconventional MHC-I MICA gene significantly reduces the incidence of acute and chronic GVHD. Blood. 2016 Oct;128(15): 1979-86.

93 Fuerst D, Neuchel C, Niederwieser D, Bunjes D, Gramatzki M, Wagner E, et al. Matching for the MICA-129 polymorphism is beneficial in unrelated hematopoietic stem cell transplantation. Blood. 2016 Dec;128(26):316976.

94 Tsamadou C, Fürst D, Vucinic V, Bunjes D, Neuchel C, Mytilineos D, et al. Human leukocyte antigen-E mismatch is associated with better hematopoietic stem cell transplantation outcome in acute leukemia patients. Haematologica. 2017 Nov;102(11):1947-55. 